\title{
Broadband inelastic light scattering of a relaxor ferroelectric $0.71 \mathrm{~Pb}\left(\mathrm{Ni}_{1 / 3} \mathrm{Nb}_{2 / 3}\right) \mathrm{O}_{3}-0.29 \mathrm{PbTiO}_{3}$
}

\author{
Shinya Tsukada, ${ }^{\text {a) }}$ Yuji lke, and Jun Kano \\ Graduate School of Pure and Applied Sciences, University of Tsukuba, Tsukuba, Ibaraki 305-8573, Japan \\ Tadashi Sekiya, Yoshihito Shimojo, and Ruiping Wang \\ National Institute of Advanced Industrial Science and Technology, Tsukuba, Ibaraki 305-8568, Japan \\ Seiji Kojima ${ }^{\text {b) }}$ \\ Graduate School of Pure and Applied Sciences, University of Tsukuba, Tsukuba, Ibaraki 305-8573, Japan
}

(Received 28 August 2006; accepted 8 October 2006; published online 21 November 2006)

\begin{abstract}
Brillouin and Raman scatterings of a $0.71 \mathrm{~Pb}\left(\mathrm{Ni}_{1 / 3} \mathrm{Nb}_{2 / 3}\right) \mathrm{O}_{3}-0.29 \mathrm{PbTiO}_{3}$ single crystal have been measured to investigate broadband inelastic spectra. The two different central peaks related to fast and slow relaxation processes have been observed separately. These two processes are attributed to the thermally activated switching of polarization in polar nanoregions. By the analysis of modified superparaelectric model, the activation energies of fast and slow relaxation processes are determined to be $3.66 \times 10^{3}$ and $4.31 \times 10^{2} \mathrm{~K}$, respectively. The fast process with the lower activation energy probably originated from $180^{\circ}$ switching, whereas the slow one with the higher energy from non- $180^{\circ}$ switching. () 2006 American Institute of Physics. [DOI: 10.1063/1.2393159]
\end{abstract}

Lead nickel niobate $\left[\mathrm{Pb}\left(\mathrm{Ni}_{1 / 3} \mathrm{Nb}_{2 / 3}\right) \mathrm{O}_{3}\right.$ or $\left.\mathrm{PNN}\right)$ is one of the oldest relaxors found by Smolensky, which exhibits a cubic to rhombohedral structural phase transition on cooling. ${ }^{1}$ Relaxor ferroelectrics have been one of the most technologically important materials because of their rich applications such as medical imaging, telecommunication, and ultrasonic devices using their huge piezoelectric and dielectric constants. ${ }^{2,3}$ The piezoelectric properties of $B$-site complex relaxor ferroelectrics, $A\left(B^{\prime} B^{\prime \prime}\right) \mathrm{O}_{3}$, are improved by adding lead titanate $\left(\mathrm{PbTiO}_{3}\right.$ or $\left.\mathrm{PT}\right)$. With adding $\mathrm{PT}$ in $A\left(B^{\prime} B^{\prime \prime}\right) \mathrm{O}_{3}$, the morphotropic phase boundary (MPB) appears and the piezoelectricity becomes the largest near MPB, where multiple phases are intricate. ${ }^{4}$ PT-doped lead magnesium niobate $\left[\mathrm{Pb}\left(\mathrm{Mg}_{1 / 3} \mathrm{Nb}_{2 / 3}\right) \mathrm{O}_{3}-\mathrm{PbTO}_{3}\right.$ or $\left.\mathrm{PMN}-\mathrm{PT}\right]$ and lead zinc niobate $\left[\mathrm{Pb}\left(\mathrm{Zn}_{1 / 3} \mathrm{Nb}_{2 / 3}\right) \mathrm{O}_{3}-\mathrm{PbTiO}_{3}\right.$ or PZN-PT] have been well studied, ${ }^{5,6}$ whereas PNN-PT has not been studied well. However, in the viewpoint of application, it has comparable piezoelectric properties to PMN-PT. ${ }^{2,7}$ One of the reasons why the study of PNN-PT was rare was the difficulty of sintering ceramics. Currently, however, some problems have been solved and several reports on its properties are available. ${ }^{2,7,8}$ Thus it is expected that the study of PNNbased relaxor ferroelectrics will be an active demand.

Common features of the ferroelectric phase transition in relaxor ferroelectrics are remarkable dielectric dispersion and central peaks (CPs) observed by Brillouin, Raman, and neutron inelastic scattering measurements. ${ }^{9-15}$ The CPs show marked temperature dependences in both width and intensity, and various kinds of mechanisms were proposed for CPs. ${ }^{16}$ In the frequency range from megahertz to terahertz in relaxor ferroelectrics, the reasonable origin of $\mathrm{CP}$ is the fluctuation of polarization related to the existence of polar nanoregion (PNR). ${ }^{10-15}$ Although PNR has been thought to play an im-

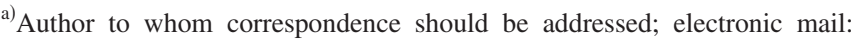
s-tsukada@ims.tsukuba.ac.jp

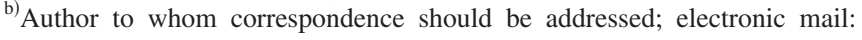
kojima@ims.tsukuba.ac.jp
}

portant role in controling the unique properties of relaxor ferroelectrics, the dynamical property of PNR has not been fully understood. In this letter, we report the broadband inelastic light scattering by the combination of Brillouin and Raman spectra of a 0.71PNN-0.29PT single crystal with near the MPB composition [ $\sim 0.32 \mathrm{PT}$ (Refs. 7 and 8 )].

The aim of this study is to investigate the dynamics of PNR by the observation of the CPs in the wide frequency spectra to clarify the nonsingle Debye nature of $\mathrm{CP}$ as $\mathrm{Na}$ kata $e t$ al. proposed recently. ${ }^{13}$ To discuss the values of the width and the intensity of CPs accurately, Brillouin and Raman scatterings were measured at the vertical-horizontal $(\mathrm{VH})$ scattering geometry where the elastic scattering is relatively weak. The unified analysis of CPs must be important because many papers on CPs in the spectra of Brillouin and Raman scatterings are discussed individually. In our knowledge, no reports discussed well the difference of CPs observed in Brillouin and Raman spectra.

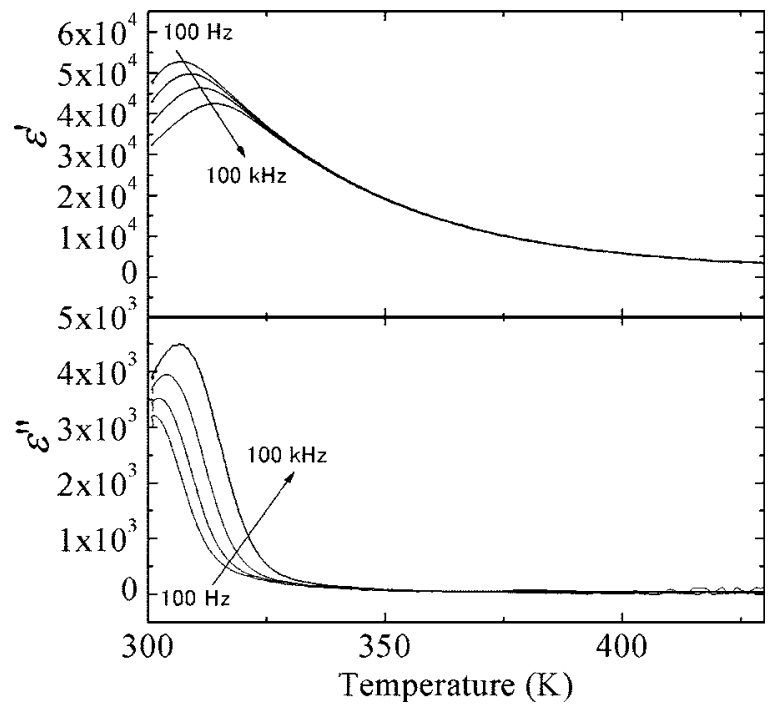

FIG. 1. Real $\left(\varepsilon^{\prime}\right)$ and imaginary $\left(\varepsilon^{\prime \prime}\right)$ parts of the dielectric permittivity of $0.71 \mathrm{PNN}-0.29 \mathrm{PT}$ as a function of temperature. 

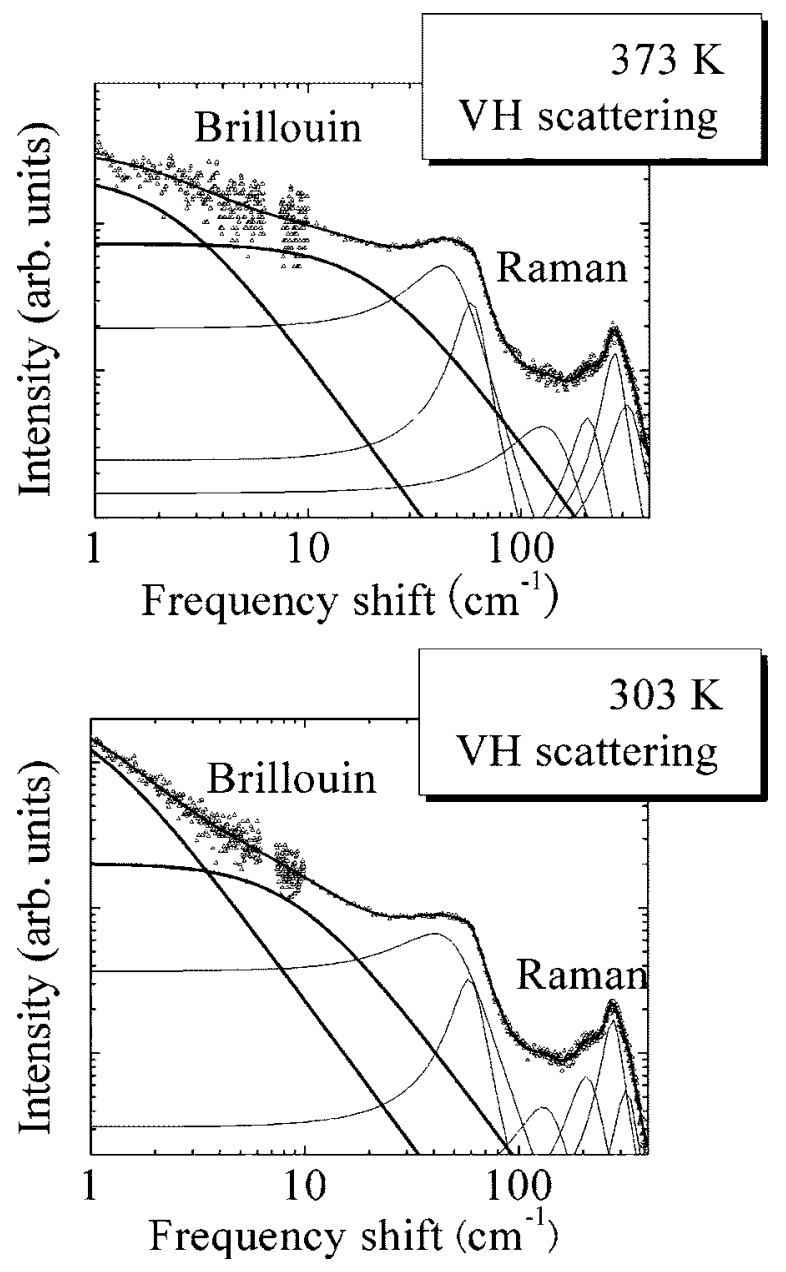

FIG. 2. Brillouin and Raman spectra of 0.71PNN-0.29PT at VH scattering geometry at 373 and $303 \mathrm{~K}$ in $\log -\log$ scale. Fitted curves constitute of two Lorentzian functions for CPs and DHOs multiplied by $(n(\omega)+1)$ for all the frequency range, where $n(\omega)$ denotes the Bose-Einstein factor.

A single crystal of $0.71 \mathrm{PNN}-0.29 \mathrm{PT}$ with light green color was prepared by the flux method. ${ }^{17}$ The concrete composition was determined by the comparison of maximum temperature $T_{m}$ of the real part of the dielectric constant with the data by Lei et al. ${ }^{8}$ Each surface was perpendicular to $\langle 100\rangle$ of the pseudocubic orientations that was confirmed by $\mathrm{x}$-ray diffraction. Brillouin spectra were measured by a high contrast $3+3$ pass tandem Fabry-Pérot interferometer combined with an optical microscope (Olympus BH-2). ${ }^{9,10}$ Raman spectra were measured by a triple grating spectrometer of additional dispersion (Jobin Yvon, T6400) with a resolution of $3 \mathrm{~cm}^{-1}$. For the temperature variation, the sample was placed inside a cryostat cell (Linkam, THMS600) with a stability of $1 \mathrm{~K}$ and an accuracy of $5 \mathrm{~K}$. The light source was a diode-pumped solid-state laser (Coherent) with a single frequency operation at $532 \mathrm{~nm}$ with $100 \mathrm{~mW}$. The scattered light was collected at the scattering angle of $180^{\circ}$. Complex dielectric permittivity was measured by an Agilent LCR meter (4294A) in the frequency range from $100 \mathrm{~Hz}$ to $1 \mathrm{MHz}$ and in the temperature range from 300 to $773 \mathrm{~K}$.

0.71PNN-0.29PT undergoes a ferroelectric phase transition from cubic to rhombohedral phases, and its rhombohedral crystal structure at room temperature was confirmed by x-ray diffraction experiments. ${ }^{7,8}$ Figure 1 shows the real $\left(\varepsilon^{\prime}\right)$ and imaginary $(\varepsilon ")$ parts of the complex dielectric permittivity of $0.71 \mathrm{PNN}-0.29 \mathrm{PT}$ measured on cooling. They show freDownloaded 30 Nov 2009 to 130.158 .56 .186 . Redistribution subject

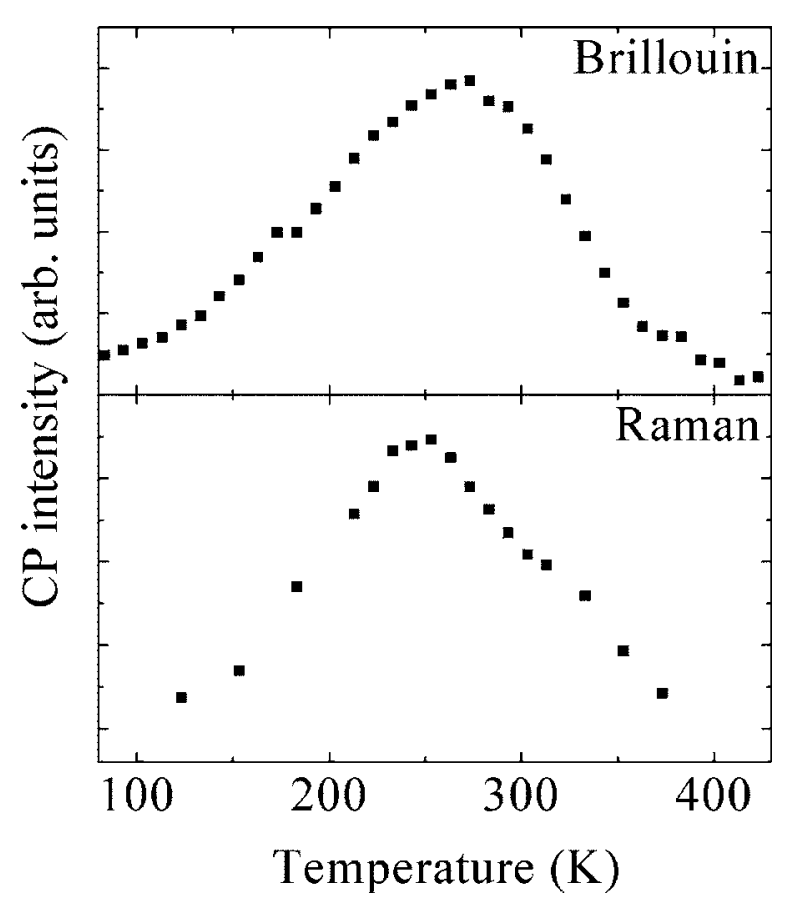

FIG. 3. Temperature dependences of the intensity of CP measured by Brillouin (upper) and Raman scatterings (lower). In Brillouin scattering measurements, FSR was $150 \mathrm{GHz}$ and the scan range was $140 \mathrm{GHz}$.

quency dispersion clearly around $T_{m}$, which is a typical relaxor behavior. The dominant contribution of this typical behavior in $\varepsilon^{\prime}$ above $T_{m}$ is believed to be both the thermally activated reorientation of dipole moments of PNR and fluctuations of PNRs boundaries. ${ }^{18}$ In general, the size of PNR is about $1-5 \mathrm{~nm},{ }^{6}$ and can be considered as an individual thermally activated switching polarization. The distribution of switching times of dipoles causes the distribution of relaxation time. ${ }^{18}$ Compared with the real part of the dielectric constant of PNN,${ }^{11}$ it can be seen that PT enhances its dielectric property and makes the diffuseness and the frequency dispersion of the phase transition smaller. $T_{m}$ was obtained at each frequency as $307.0 \mathrm{~K}$ at $100 \mathrm{kHz}, 309.1 \mathrm{~K}$ at $10 \mathrm{kHz}$, $311.1 \mathrm{~K}$ at $1 \mathrm{kHz}$, and $313.8 \mathrm{~K}$ at $100 \mathrm{~Hz}$.

The Brillouin and Raman spectra of 0.71PNN-0.29PT in VH scattering geometry are shown in Fig. 2. In order to obtain the broadband light scattering spectra from $1 \mathrm{~cm}^{-1}$ $(=30 \mathrm{GHz})$ to $1000 \mathrm{~cm}^{-1}$, the Brillouin spectra with the free spectral range (FSR), $200 \mathrm{GHz}$, and the scanning range, $300 \mathrm{GHz}$, were combined with Raman spectra above $10 \mathrm{~cm}^{-1}$ in several temperatures. As usual, CP is analyzed by a Lorentzian function centered at zero frequency shift assuming a single relaxation. ${ }^{10-12,14,15}$ Independent damped harmonic oscillators (DHOs) were used for all the phonon modes. ${ }^{14,15}$ In the broadband spectra, it is necessary to analyze using two Lorentzians, which correspond to two relaxation processes related to PNRs. The polarization in PNRs along the eight equivalent $\langle 111\rangle$ directions in the pseudocubic structure was determined by means of dynamical structural analysis of diffuse neutron scattering in PMN. ${ }^{6}$ In the case of polarization switching in barium titanate, the $180^{\circ}$ domain can switch faster than the $90^{\circ}$ domain because no strain is induced during switching. ${ }^{19}$ In other words, the energy barrier of $180^{\circ}$ domain switching can be much smaller than that of the $90^{\circ}$ domain switching. A similar situation occurs for the switching of polarization of PNRs in to AlP license or copyright; see http://apl.aip.org/apl/copyright.jsp 


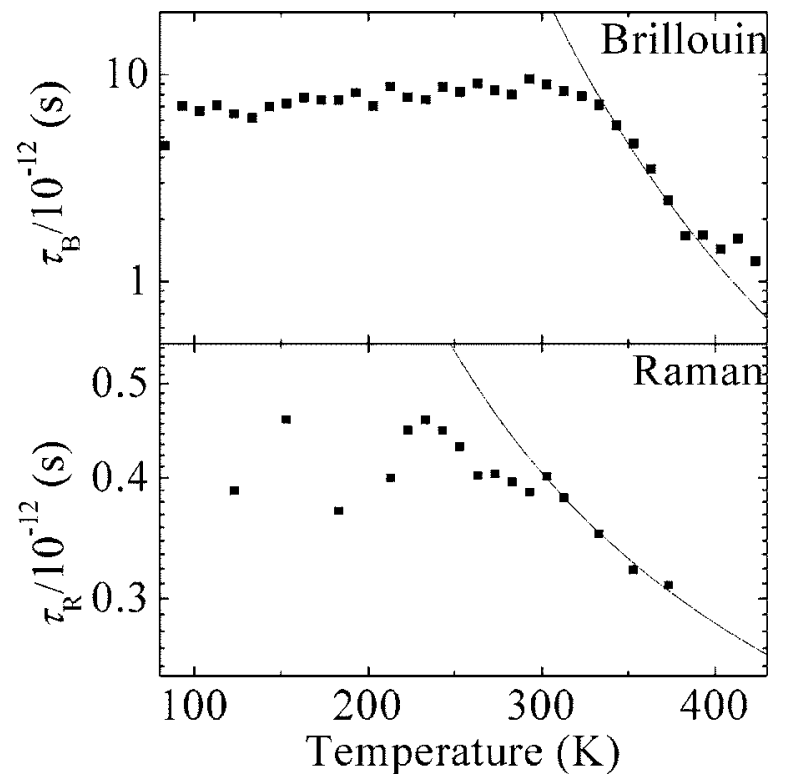

FIG. 4. Temperature dependences of the two relaxation times obtained by FWHM of CP on a semilogarithmic plot for Brillouin (upper: $\tau_{B}$ ) and Raman (lower: $\tau_{R}$ ) scatterings. The solid lines are fitted results by the modified superparaelectric model.

0.71PNN-0.29PT, and two relaxations in the dipole switching can be considered in PNRs. The slower relaxation (obtained in Brillouin spectra) can correspond to the non- $180^{\circ}$ switching process followed by the change of strain, while the faster relaxation (obtained in Raman spectra) can correspond to the $180^{\circ}$ switching free from any change of strain.

Figure 3 shows temperature dependences of the intensity of CPs observed by Brillouin and Raman scatterings, respectively. The intensity of CPs increases on cooling, which indicates that the number density of PNRs increases. The maxima in the temperature dependence of the intensity of CPs are $270 \mathrm{~K}$ for the slower relaxation and $250 \mathrm{~K}$ for the faster one. We consider that these maximum temperatures are the freezing temperature $T_{f}$, from which the switching process of the PNRs begins to freeze on cooling. ${ }^{20}$ The difference between the two CPs is explained by the difference of potential barriers to switch the polarization in PNRs as discussed above.

To determine the potential barriers, the relaxation time $\tau$ was obtained by the equation $\tau=1 / \pi \Delta \Gamma$, where $\Delta \Gamma$ is the full width at half maximum (FWHM) of CP. The obtained temperature dependences of the two relaxation times and the intensity are shown in Fig. 4. By the analysis of the modified superparaelectric model, ${ }^{10}$ the activation energies extrapolated to $0 \mathrm{~K}, H_{0}$, of fast and slow relaxation processes are determined to be $H_{0 B} \sim 3.66 \times 10^{3} \mathrm{~K}$ for CPs in the Brillouin spectra and $H_{0 R} \sim 4.31 \times 10^{2} \mathrm{~K}$ for CPs in the Raman spectra. $H_{0 B}$ and $H_{0 R}$ probably correspond to the potential barrier of non- $180^{\circ}$ polarization switching and that of $180^{\circ}$ polariza- tion switching, respectively. The value of observed $H_{0 B}$ is reasonable in comparison with the previous works, for example, $H_{0 B} \sim 6136 \mathrm{~K}$ for $0.65 \mathrm{PMN}-0.35 \mathrm{PT},{ }^{10} H_{0 B} \sim 1309 \mathrm{~K}$ for PNN, ${ }^{11} H_{0 B} \sim 1900 \mathrm{~K}$ for PZN. ${ }^{12} H_{0 R}$ determined, however, cannot be compared with other data in literatures.

We have reported dielectric, Brillouin, and Raman scattering studies of a $0.71 \mathrm{PNN}-0.29 \mathrm{PT}$ single crystal focusing on CP. The comparison between CPs observed in Brillouin and Raman spectra indicates that the relaxation times of each $\mathrm{CP}$ are different. By the analysis of the modified superparaelectric model for the two CPs, we obtained the activation energy $H_{0 B} \sim 3.66 \times 10^{3} \mathrm{~K}$ for CPs in Brillouin spectra and $H_{0 R} \sim 4.31 \times 10^{2} \mathrm{~K}$ for Raman spectra. These results indicate that the different origins in CPs observed in Brillouin and Raman spectra are attributed to the two characteristic relaxation processes of polarization switchings in polar nanoregions, i.e., non- $180^{\circ}$ switching (slower) and $180^{\circ}$ switching (faster). To confirm the interpretation on the two different $\mathrm{CPs}$, further experiments are required. The spectra measured at the vertical-vertical scattering geometry and the other modes in the VH scattering geometry will be discussed separately in the next paper.

${ }^{1}$ G. A. Smolensky, J. Phys. Soc. Jpn 28, 26 (1970).

${ }^{2}$ S.-E. Park and T. R. Shrout, J. Appl. Phys. 82, 1804 (1997).

${ }^{3}$ D.-S. Paik, S.-E. Park, T. R. Shrout, S. Wada, S.-F. Liu, and T. R. Shrout, J. Appl. Phys. 85, 1080 (1999).

${ }^{4}$ B. Noheda, D. E. Cox, G. Shirane, J. Gao, and Z.-G. Ye, Phys. Rev. B 66, 054104 (2002).

${ }^{5}$ Z.-G. Ye, Key Eng. Mater. 155, 81 (1998).

${ }^{6}$ A. A. Bokov and Z.-G. Ye, J. Mater. Sci. 41, 31 (2006).

${ }^{7}$ K. Kusumoto and T. Sekiya, Ferroelectrics 240, 327 (2000).

${ }^{8}$ C. Lei, K. Chen, X. Zhang, and J. Wang, Solid State Commun. 123, 445 (2002).

${ }^{9}$ D. H. Kim, J.-H. Ko, C. D. Feng, and S. Kojima, J. Appl. Phys. 98, 044106 (2005).

${ }^{10}$ F. M. Jang and S. Kojima, Phys. Rev. B 62, 8572 (2000).

${ }^{11}$ J. Fan, M. H. Kuok, S. C. Ng, N. Yasuda, H. Ohwa, H. Orihara, H. M. Iwata, and Y. Ishibashi, J. Appl. Phys. 91, 2262 (2002).

${ }^{12}$ Y. Gorouya, Y. Tsujimi, M. Iwata, and T. Yagi, Appl. Phys. Lett. 83, 1358 (2003).

${ }^{13}$ Y. Nakata, Y. Tsujimi, K. Katsuraya, M. Iwata, and T. Yagi, Appl. Phys. Lett. 89, 022903 (2006).

${ }^{14}$ J. Toulous, F. Jang, O. Svitelskiy, W. Chen, and Z.-G. Ye, Phys. Rev. B 72, 184106 (2005).

${ }^{15}$ I. G. Siny, S. G. Lushnikov, and R. S. Katiyar, Phys. Rev. B 56, 7962 (1997).

${ }^{16} \mathrm{P}$. A. Fleury and K. B. Lyons, Modern Problems in Condensed Matter Sciences 5, 449 (1983).

${ }^{17}$ T. Sekiya, K. Kusumoto, H. J. Hwang, J. P. Reyes, J. P. Chaminade, and J. Ravez, J. Korean Phys. Soc. 32, S1201 (1998).

${ }^{18}$ V. Bovtun, J. Petzelt, V. Porokhonskyy, S. Kamba, and Y. Yakimenko, J. Eur. Ceram. Soc. 21, 1307 (2001).

${ }^{19}$ S. Wada, S. Suzuki, T. Noma, T. Suzuki, M. Osada, M. Kakihana, S.-E. Park, L. E. Gross, and T. R. Shrout, Jpn. J. Appl. Phys., Part 1 38, 5505 (1999).

${ }^{20}$ G. Xu, G. Shirane, J. R. D. Copley, and P. M. Gehring, Phys. Rev. B 69, 064112 (2004) 